Ji Min Kim¹, Sorim Choung², Sun Mi Kang ${ }^{3}$, Younghak Lee', Yea Eun Kang1, Hyon-Seung Yi', Kyong-Hye Joung', Ju Hee Lee', Hyun Jin Kim ${ }^{1}$, Bon Jeong Ku, ${ }^{1}$

\begin{abstract}
${ }^{1}$ Department of Internal Medicine, Chungnam National University School of Medicine, Daejeon, Republic of Korea ${ }^{2}$ Department of Medical Science Medicine, Chungnam National University School of Medicine, Daejeon, Republic of Korea ${ }^{3}$ Department of Endocrinology \& Metabolism, Sun General Hospital, Daejeon, Republic of Korea
\end{abstract}

\begin{abstract}
Objective
Soluble Leucine rich repeats and immunoglobulin like domains 2 (LRIG2) interact with epidermal growth factor receptor (EGFR), resulting in activation of EGFR and its downstream pathways. We previously reported the association of serum EGFR with type 2 diabetes mellitus (T2DM), and LRIG2 is also expected to be correlates with T2DM.
\end{abstract}

\section{Aim of Study}

The purpose of current study was to compare soluble LRIG2 level in T2DM and controls, and to investigate the relationship between soluble LRIG2 and glucose parameters.

\section{Methods}

A total of $\mathbf{2 4 0}$ subjects were enrolled in this study, and they were divided into three groups [ 80 with T2DM, 80 with prediabetes and 80 with normoglycemia (NGT)]. Blood samples were collected in the morning after an overnight fast $>8$ hours. Fasting (FBS) and 2-hour postprandial (PP2) plasma glucose levels, fasting insulin and C-peptide levels were measured. Fasting serum LRIG2 levels were measured using a quantitative sandwich enzyme immunoassay technique with an enzyme-linked immunosorbent assay kit (MyBioSource, China; catalog no. MBS9337066). Demographic findings and serum LRIG2 were compared among three groups, and serum LRIG2 were correlated with various clinical factors using linear regression analysis.

\begin{tabular}{|c|c|c|c|c|}
\hline \multicolumn{5}{|c|}{ Results } \\
\hline & $\begin{array}{c}\text { NGT } \\
(n=80)\end{array}$ & $\begin{array}{l}\text { Prediabetes } \\
(\mathrm{n}=80)\end{array}$ & $\begin{array}{l}\text { T2DM } \\
(n=80)\end{array}$ & $\mathrm{p}$-value \\
\hline Age (mean \pm SD, years) & $50.7 \pm 13.7$ & $53.5 \pm 12.1$ & $54 \pm 12.3$ & 0.210 \\
\hline Sex (male/female) & $25 / 55$ & $38 / 42$ & $35 / 45$ & 0.091 \\
\hline $\operatorname{HTN}(n, \%)$ & & $14(17.5)$ & $33(41.3)$ & \\
\hline Smoking (n, \%) & $8(10.0)$ & $7(8.8)$ & $22(27.5)$ & 0.001 \\
\hline Height (mean $\pm \mathrm{SD}, \mathrm{cm}$ ) & $161.6 \pm 8.6$ & $163.2 \pm 8.4$ & $163.3 \pm 9.4$ & 0.528 \\
\hline Weight (mean \pm SD, kg) & $63.9 \pm 12.4$ & $67.1 \pm 11.9$ & $70.1 \pm 14.7$ & 0.039 \\
\hline BMI (mean $\left.\pm \mathrm{SD}, \mathrm{kg} / \mathrm{m}^{2}\right)$ & $24.3 \pm 3.3$ & $24.8 \pm 3.2$ & $26.2 \pm 4.4$ & 0.020 \\
\hline $\mathrm{SBP}($ mean $\pm \mathrm{SD}, \mathrm{mmHg})$ & $127.8 \pm 16.1$ & $128.9 \pm 14.6$ & $134.6 \pm 20.2$ & 0.065 \\
\hline $\mathrm{DBP}($ mean $\pm \mathrm{SD}, \mathrm{mmHg})$ & $79.3 \pm 12.6$ & $77.4 \pm 10.9$ & $81.8 \pm 11.8$ & 0.102 \\
\hline $\mathrm{Hb}($ mean $\pm \mathrm{SD}, \mathrm{g} / \mathrm{dL})$ & $13.7 \pm 1.2$ & $14.1 \pm 1.7$ & $14.5 \pm 1.5$ & 0.015 \\
\hline HbA1c (mean \pm SD, \%) & $5.3 \pm 0.2$ & $5.6 \pm 0.3$ & $7.4 \pm 1.9$ & 0.000 \\
\hline FBS (mean \pm SD, mg/dL) & $91.9 \pm 5$ & $101.7 \pm 11.1$ & $154.2 \pm 57.3$ & 0.000 \\
\hline PP2 (mean $\pm \mathrm{SD}, \mathrm{mg} / \mathrm{dL}$ ) & $105.3 \pm 19$ & $143.3 \pm 33.5$ & $293 \pm 101.2$ & 0.000 \\
\hline Insulin, fasting (mean $\pm \mathrm{SD}, \mathrm{mg} / \mathrm{dL}$ ) & $8.3 \pm 4.2$ & $10 \pm 5$ & $18 \pm 38.9$ & 0.019 \\
\hline Insulin, PP2 (mean $\pm \mathrm{SD}, \mathrm{mg} / \mathrm{dL}$ ) & $36.6 \pm 43.6$ & $68.9 \pm 68$ & $70.7 \pm 69.5$ & 0.001 \\
\hline C-peptide, fasting (mean $\pm \mathrm{S}$ & $0.73 \pm 0.47$ & $0.87 \pm 0.5$ & $1.15 \pm 0.71$ & 0.000 \\
\hline C-peptide, PP2 (mean $\pm \mathrm{SD}, \mathrm{ng} / \mathrm{mL}$ ) & $3.44 \pm 2.23$ & $4.09 \pm 2.7$ & $6.72 \pm 24.34$ & 0.347 \\
\hline $\mathrm{TG}($ mean $\pm \mathrm{SD}, \mathrm{mg} / \mathrm{dL})$ & $104 \pm 63.2$ & $145.1 \pm 106.7$ & $189.6 \pm 173$ & 0.000 \\
\hline Tchol (mean $\pm \mathrm{SD}, \mathrm{mg} / \mathrm{dL}$ ) & $191.4 \pm 30.4$ & $196.6 \pm 39.8$ & $192.3 \pm 46.9$ & 0.682 \\
\hline $\mathrm{HDL}($ mean $\pm \mathrm{SD}, \mathrm{mg} / \mathrm{dL}$ ) & $61.7 \pm 13.7$ & $53.7 \pm 14$ & $49 \pm 13.4$ & 0.000 \\
\hline $\mathrm{LDL}$ (mean $\pm \mathrm{SD}, \mathrm{mg} / \mathrm{dL}$ ) & $113.5 \pm 29.7$ & $121.5 \pm 34.2$ & $116.6 \pm 45.3$ & 0.389 \\
\hline $\mathrm{BUN}($ mean $\pm \mathrm{SD}, \mathrm{mg} / \mathrm{dL}$ ) & $13.7 \pm 3.6$ & $14 \pm 3.7$ & $14.8 \pm 4.6$ & 0.206 \\
\hline & $0.73 \pm 0.15$ & $0.75 \pm 0.18$ & $0.72 \pm 0.19$ & 0.496 \\
\hline eGFR (mean $\left.\pm \mathrm{SD}, \mathrm{mL} / \mathrm{min} / 1.73 \mathrm{~m}^{2}\right)$ & $103.4 \pm 18.1$ & $104.2 \pm 24.4$ & $112 \pm 29.4$ & 0.061 \\
\hline AST (mean \pm SD, U/L) & $21.3 \pm 6.6$ & $23.2 \pm 11.4$ & $26.5 \pm 14.9$ & 0.019 \\
\hline $\mathrm{ALT}($ mean $\pm \mathrm{SD}, \mathrm{U} / \mathrm{L})$ & $20.7 \pm 15$ & $25 \pm 20.8$ & $29.1 \pm 16.8$ & 0.017 \\
\hline
\end{tabular}

* The $p$-value was obtained from student t-test

$\dagger$ The $p$-value was obtained from the chi-squared test.

In baseline characteristic analysis, significant difference among the three groups were found in HTN $(p=0.001)$, smoking $(p=0.001)$, weight $(p=0.039), B M I(p=0.020), H b(p=0.015), H b A 1 c(p<0.001)$, fasting glucose $(p<0.001), P P 2(p<0.001)$, fasting insulin $(p=0.019), P P 2$ insulin $(p=0.001)$, fasting $C$-peptide $(p<0.001)$, TG $(p<0.001)$, HDL-C $(p<0.001)$, AST $(p=0.019)$, and ALT $(p=0.017)$. There was no difference in the other factors including age, sex, height, and BP (all $p>0.05$ ) (Table 1 ).
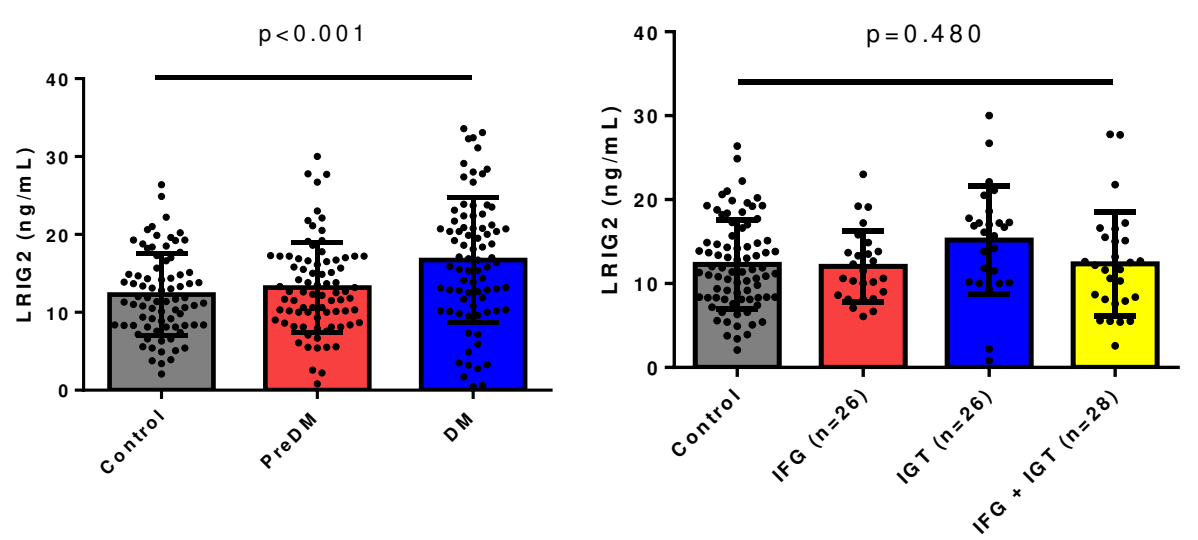

Figure 1. Comparison of Soluble LRIG2 levels
Serum LRIG2 levels in T2DM group $(16.73 \pm 8.01 \mathrm{ng} / \mathrm{mL})$ was significantly higher than those of preDM $(13.18 \pm 5.83 \mathrm{ng} / \mathrm{mL}, \mathrm{p}=0.002)$ and control group $(12.29 \pm 5.27 \mathrm{ng} / \mathrm{mL}, p<0.001)$. For subgroup analysis, preDM group was divided into 3 groups as impaired fasting glucose (IFG, $n=26$ ), impaired glucose tolerance (IGT, $n=26)$, and IFG + IGT $(n=28)$. There were no significant differences in serum LRIG2 levels between groups $(p=0.099)$ (Fig. 1).

Table 2. Correlation between LRIG2 and clinical factors

\begin{tabular}{|c|c|c|c|c|c|c|c|c|}
\hline \multirow[b]{2}{*}{ Age } & \multicolumn{2}{|c|}{ Total } & \multicolumn{2}{|c|}{ NGT } & \multicolumn{2}{|c|}{ PreDM } & \multicolumn{2}{|c|}{ T2DM } \\
\hline & $\begin{array}{c}\frac{r}{-0.065} \\
\end{array}$ & $\frac{p}{0.317}$ & $\begin{array}{c}r \\
0.122\end{array}$ & $\frac{p}{0.280}$ & $\frac{r}{-0.160}$ & $\frac{p}{0.157}$ & $\begin{array}{c}r \\
-0.211\end{array}$ & $\frac{p}{0.060}$ \\
\hline Height & 0.122 & 0.103 & 0.035 & 0.806 & -0.113 & 0.422 & 0.247 & 0.034 \\
\hline weight & 0.175 & 0.019 & -0.057 & 0.690 & 0.043 & 0.759 & 0.239 & 0.040 \\
\hline BMI & 0.139 & 0.065 & -0.107 & 0.454 & 0.090 & 0.525 & 0.146 & 0.219 \\
\hline SBP & 0.179 & 0.016 & 0.270 & 0.055 & -0.088 & 0.528 & 0.185 & 0.114 \\
\hline DBP & 0.216 & 0.004 & 0.236 & 0.096 & 0.153 & 0.269 & 0.197 & 0.092 \\
\hline $\mathrm{Hb}$ & 0.109 & 0.128 & -0.006 & 0.962 & 0.006 & 0.966 & 0.110 & 0.396 \\
\hline HbA1c & 0.305 & 0.000 & 0.021 & 0.856 & 0.060 & 0.603 & 0.239 & 0.037 \\
\hline FBS & 0.319 & 0.000 & -0.068 & 0.547 & -0.181 & 0.107 & 0.298 & 0.007 \\
\hline PP2 & 0.320 & 0.000 & 0.157 & 0.165 & 0.167 & 0.138 & 0.181 & 0.109 \\
\hline Insulin, FBS & 0.073 & 0.260 & -0.224 & 0.047 & -0.062 & 0.590 & 0.051 & 0.656 \\
\hline Insulin, PP2 & -0.004 & 0.955 & -0.065 & 0.589 & 0.078 & 0.519 & -0.146 & 0.198 \\
\hline C-peptide, FBS & 0.144 & 0.034 & 0.014 & 0.909 & 0.090 & 0.453 & 0.061 & 0.603 \\
\hline C-peptide, PP2 & -0.001 & 0.988 & 0.107 & 0.376 & 0.136 & 0.259 & -0.062 & 0.606 \\
\hline TG & 0.176 & 0.007 & 0.070 & 0.540 & -0.001 & 0.995 & 0.168 & 0.153 \\
\hline TC & -0.024 & 0.715 & -0.086 & 0.452 & -0.132 & 0.253 & 0.075 & 0.526 \\
\hline HDL-C & -0.125 & 0.059 & -0.253 & 0.025 & 0.027 & 0.814 & 0.067 & 0.574 \\
\hline LDL-C & -0.074 & 0.264 & 0.003 & 0.978 & -0.164 & 0.154 & -0.071 & 0.555 \\
\hline BUN & 0.101 & 0.131 & 0.005 & 0.963 & 0.088 & 0.449 & 0.093 & 0.441 \\
\hline $\mathrm{Cr}$ & 0.096 & 0.152 & 0.096 & 0.401 & 0.087 & 0.453 & 0.145 & 0.228 \\
\hline eGFR & 0.033 & 0.623 & -0.102 & 0.370 & -0.002 & 0.984 & 0.013 & 0.914 \\
\hline AST & 0.118 & 0.076 & -0.068 & 0.553 & -0.023 & 0.844 & 0.167 & 0.165 \\
\hline ALT & 0.113 & 0.089 & -0.056 & 0.625 & 0.106 & 0.359 & 0.108 & 0.369 \\
\hline
\end{tabular}

In pearson correlation analysis, serum LRIG2 was significantly correlated with HbA1c $(r=-0.305, p<0.001)$, FBS $(r=0.319, p<0.001)$ and PP2 $(r=0.320$, $\mathrm{p}<0.001$ ) (Table 2).
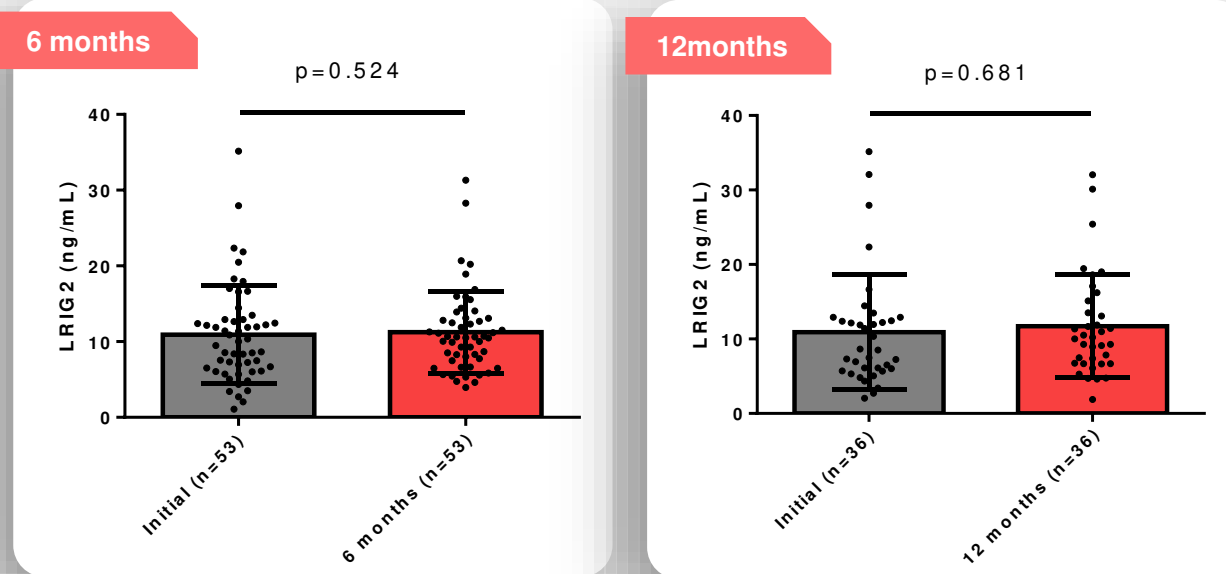

Figure 2. Change of LRIG2 levels after metformin treatment.

Table 3. Association between serum LRIG2 change and change in clinical factors

\begin{tabular}{lcc}
\hline & $r$ & $p$-value \\
\hline HbA1c changes & -0.006 & 0.967 \\
FBS changes & 0.265 & 0.431 \\
Weight changes & 0.076 & 0.609 \\
BMI changes & 0.063 & 0.669 \\
TG changes & -0.072 & 0.666 \\
TC changes & -0.132 & 0.356 \\
HDL changes & -0.124 & 0.386 \\
LDL changes & 0.084 & 0.553 \\
\hline
\end{tabular}

A total of 53 subjects with T2DM were additionally enrolled and treated with metformin monotherapy. Serum LRIG2 levels before $(10.90 \pm 9.50)$ and after 6 months $(11.25 \pm 5.42)$ of treatment did not show any significant difference $(n=53, p=0.524)$. And these trends were also obtained in the 31 subjects who were followed up 1-year (11.59 \pm 6.38 , $\mathrm{p}=0.681$ ) (Fig. 2). In addition, no significant correlation was found between the changes of LRIG2 and clinical factors (Table 3 ).

\section{Conclusion}

Serum LRIG2 levels were highest in the T2DM group and higher in the prediabetes group than in the NGT group. And there were no significant changes in serum LRIG2 levels after 6 or 12 months of metformin treatment. The results of our study suggest a possible assosiation of EGFR signaling with T2DM and serum LRIG2 might be a novel biomarker in diagnosis of T2DM. 